\title{
Cubosomes: An Overview
}

\author{
Gopal GARG, * Shailendra SARAF, and Swarnlata SARAF \\ Institute of Pharmacy, Pt. Ravishankar Shukla University; Raipur, 492010, India.
}

Received August 20, 2006; accepted October 29, 2006

Cubosomes are nanoparticles but instead of the solid particles, cubosomes are self-assembled liquid crystalline particles of certain surfactant with proper ratio of water with a microstructure that provides unique properties of practical interest. The discovery of cubosomes is a unique story and spans the field of food science, differential geometry, biological membranes and digestive processes. One of the most common surfactants used to make cubosomes is the monoglyceride glycerol monoolein. Bicontinuous cubic liquid crystalline phase is an optically clear, very viscous material that has a unique structure at the nanometer scale. The word bicontinuous refers to the division of the two continuous but non-intersecting aqueous regions by a lipid bilayer that is contorted into a space-filling structure. Hydrating a surfactant or polar lipid that forms cubic phase and then dispersing the solid-like phase into smaller particles usually form Cubosomes. There is a lot of excitement about the cubic phases because its unique microstructure is biologically compatible and capable of controlled release of solubilized active ingredients like drugs and proteins.

Key words cubosome; nanoparticles; bicontinuous; bilayer; monoolein; periodontal

Surfactant and polymers are generally used in the controlled drug delivery systems. Surfactant and polymer systems form supra-assemblies, which are extensively exploited as active delivery vehicles. These systems include liquid crystalline aggregates (e.g., liposomes and cubosomes) or cross-linked gel networks (hydrogels) that load, stabilize, and eventually deliver active ingredients. The potential for utilizing a particular active with a vehicle depends on the physicochemical properties of both. To achieve therapeutic effects, it must be possible to load sufficient amounts of the active, which largely depends on the interaction of the vehicle and active. Further, the integrity of the active must be retained through all stages: preparation, storage, and use. The release rate of actives must be controlled to achieve optimal drug release profiles, while ease of preparation and vehicle stability must also be considered. An optimal delivery vehicle must successfully encompass all these properties.

Cubosomes are bicontinuous cubic phase liquid crystals have many properties that make them appealing as a universal vehicle for drug delivery. Luzzati et al. ${ }^{1)}$ first documented its geometric model supplied later by Scriven. ${ }^{2)}$ Which is in the past decade have been examined for drug delivery. ${ }^{3)}$ The surfactant assembles into bilayers that are twisted into a three dimension, periodic, minimal surface forming tightly packed structure, like "honeycombed" with bicontinuous domains of water and lipid.

Cubosome particles are first prepared by mechanical fragmentation of the cubic lipid-water phase in a three-phase region containing a liposomal dispersion and to differentiate from liposomes, these particles have been termed as cubo- somes. ${ }^{4-8)}$ Its structure is different from liposomes because its structure can simultaneously accommodate water-soluble, lipid-soluble, and amphiphilic molecules.

Three structure of cubosomes have been proposed by Luzzati et al. ${ }^{9)}$; (i) Pn3m (D-surface) (Diamond surface), (ii) I $a 3 d$ (G-surface) (Gyroid surface), and (iii) I $m 3 m$ (P-surface) (Primitive surface), in terms of nodal surfaces.

The structure generally maintains the efficacy; stability of actives such as vitamins ${ }^{10)}$ and proteins. ${ }^{11)}$ Cubosomes are thermodynamically stable; lasting indefinitely. ${ }^{12)}$ Colloidal dispersions of cubosomes can be stabilized by the addition of polymers. ${ }^{13)}$ They also possess the potential for controlled delivery of actives, where diffusion is governed by the tortuous diffusion of the active through the "regular" channel structure of the cubic phase. ${ }^{14)}$ Cubosomes possess a sufficient average degree of molecular orientation order to characterize by structural symmetry, and often form in aqueous surfactant system at relatively high ampiphile concentrations.

History Luzzati and Husson ${ }^{15)}$ and Luzzati et al. first recognized the existence of cubic phases in lipid-water system using X-ray scattering measurement. Fontell et al. ${ }^{16)}$ drew similar conclusions regarding cubic phase in ternary systems of ampiphiles, oils and water in parallel although without apparent awareness of the lipid work. Around the same time, Lutton published a comprehensive study of the aqueous phase behavior of monoglycerides. Monoglycerides are polar lipids with poor water solubility that exhibit aqueous phase behavior reflecting their structural similarity to non-ionic surfactants. ${ }^{17)}$ Landh and Larsson have patented the preparation of colloidal dispersions of nonlamellar ly-

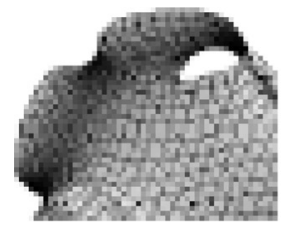

P-Surface

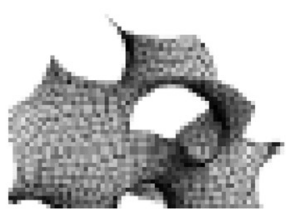

G-Surface

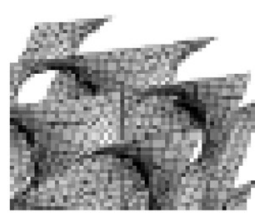

D-Surface

Fig. 1. Structure of Different Cubosomes

* To whom correspondence should be addressed. e-mail: rsofiop_gg@rediffmail.com 


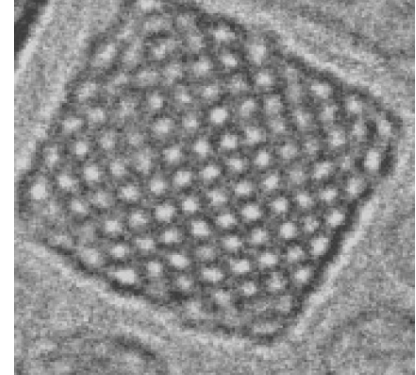

Fig. 2. Figure of Cubosomes

otropic crystalline phases and have termed the particles "cubosomes" ${ }^{18)}$ Cubosomes usually have been produced by means of time-consuming methods involving high-energy input. For instance Gustafsson et al. have investigated the production and structure of aqueous dispersions of lipidbased lyotropic liquid crystalline phases. The dispersions were based either on glycerylmonooleate/sunflower oil or glycerylmonooleate/retynilpalmitate mixtures plus a nonionic triblock polymer (Poloxamer 407) in water. Dispersions were produced by drop wise addition of a melt of lipids and poloxamer in water, followed by reduction of size by homogenization under high pressures at $80^{\circ} \mathrm{C}$. Recently Siekmann et al. ${ }^{19)}$ have reported the preparation and characterization of dispersions constituted of monoolein-rich monoglycerides with or without purified soya phospholipids Dispersions were prepared by equilibration of the monoglyceride/phospholipid/water cubic phase, subsequent fragmentation by a solution of Poloxamer 407, predispersing by probe sonication and finally high pressure homogenization. Moreover some authors have developed experimental protocols for cubosome production based on the use of organic solvents. In particular Spicer and Hyden have proposed a method based on a dilution process of an ethanolic solution of monoolein with an aqueous solution of poloxamer. Ethanol was used as a hydrotrope to create a liquid precursor, spontaneously forming cubosomes after dilution. Finally Nakano et al. have suggested a method for the production of cubosomes based on hydration of a dry film of monoolein/poloxamer with an aqueous buffer. ${ }^{20)}$

Method of Preparation of Cubosomes Three macroscopic forms of cubic phase are typically encountered; precursor, bulk gel and particulate dispersion. The precursor form exists as a solid or liquid material that forms cubic phase in response to a stimulus, such as contact with liquid. Bulk cubic phase gel is an optically isotropic, stiff, and solid like material in equilibrium with water can be dispersed into particles called cubosomes. The production of cubosomes entails two distinct technologies:

(i) Top-Down Technique: Top-down approach begins with a suitable starting material and then sculpts the functionality from the material. Most cubosome research over the last two decades has focused on top-down techniques, whereby bulk cubic phase is first produced and then dispersed by high energy processing into cubosome nanoparticles. Bulk cubic phase resembles a clear rigid gel formed by water-swollen cross-linked polymer chains, but cubic phases differ in that they are a single thermodynamic phase and display periodic liquid crystalline structure. Rupture of these cubic phases occurs in a direction parallel to the shear direction and the en-

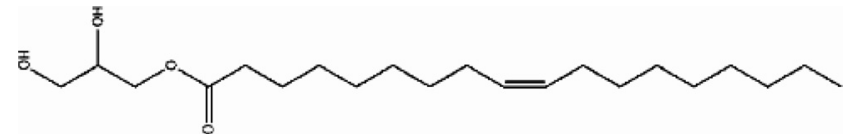

Fig. 3. Monoolein

ergy required is proportional to the number of tubular network branches that rupture. Warr and Chen found that rupture of the cubic phase occurs as the bilayer breaks under applied shear stresses and flows along slip planes. The cubic phases exhibit a yield stress that increases with increasing amounts of bilayer-forming surfactants and oils, and that is inversely proportional to the crystalline unit cell dimension. Warr and Chen also suggested but could not confirm that cubic phases may behave as lamellar phases do during dispersion with increasing shear: dispersed liquid crystalline particles form at intermediate shear rates, whereas a defectfree bulk phase re-forms at higher shear rates. At high oscillatory frequencies, cubic phases become highly elastic. ${ }^{21)}$

(ii) Bottom-Up Technique: The bottom-up approach first forms the nanostructure building blocks and then assembles them into the final material. It is more recently developed technique of cubosome formation, allowing cubosomes to form and crystallize from precursors on the molecular length scale. Almgren et al. ${ }^{22)}$ discuss the formation of cubosomes by dispersion of L2 or inverse micellar phase droplets in water at $80^{\circ} \mathrm{C}$, then by slow cooling to allow the droplets to gradually crystallize into cubosomes.

Dispersion of the nanoparticles produced in the cubosomes formation by several techniques

- Sonication

- High pressure homogenization

- Spontaneous emulsification

- Spray drying

Sonication and high-pressure homogenization suggests the formation of complex dispersions containing vesicles and cubosomes with time-dependent ratios of each particle type. Coarse cubosomes on the micron scale possess the same Dsurface cubic structure as their originating bulk cubic phase $^{23)}$ but after homogenization, the P-surface dominates, either because of the added polymer or other factors. Largescale production of cubosomes and products containing them requires more robust processes. Spicer et al. developed a room temperature process to produce cubosomes, diluting monoolein-ethanol (or other hydrotrope) solutions with aqueous Poloxamer 407 solutions by spontaneous emulsification, producing nano-particle dispersions in the near absence of energy input beyond that required for simple blending of the two liquids. Smaller and more stable cubosomes are produced than those by high-energy processes, but some vesicles are also produced. A second process was also developed to allow cubosome production from a powdered precursor. ${ }^{24)}$ Spray-dried powders comprising monoolein coated with starch or dextran form cubosomes on simple hydration. The polymers immediately provide colloidal stabilization of the cubosomes.

Material Used in Cubosomes Formation Bicontinuous cubic phases are found in natural lipids, cationic ${ }^{25)}$ and nonionic surfactants, ${ }^{26)}$ and polymer systems, although the lipid most widely used to construct bicontinuous cubic phases is the monoglyceride monoolein, monoglycerides sponta- 
neously form bicontinuous cubic phases upon the addition of water, are relatively insoluble (allowing the formation of colloidal dispersions of cubosomes), and are resistant to changes in temperature.

The main precursor of cubosome formation is monoolein. Monoolein or glyceryl monooleate is a mixture of the glycerides of oleic acid and other fatty acids, consisting mainly of the monooleate. The acyl chain is attached to the glycerol backbone by an ester bond. ${ }^{27-29)}$

The two remaining carbons of the glycerol have active hydroxyl groups, giving polar characteristics to this portion of the molecule. The glycerol moiety may form hydrogen bonds with water in an aqueous environment and is commonly referred to as the head group. The hydrocarbon chain gives hydrophobic characteristics to monoolein and is often termed the tail. ${ }^{30)}$ Commercially available monoolein may be obtained in two forms, a mixed glyceride form or as distilled monoolein; the distilled monoolein is preferred for pharmaceutical applications because of its high purity. Monoolein occurs as a waxy yellow paste with a characteristic odor. It swells in water, giving rise to several lyotropic liquid crystalline structures. From a pharmaceutical standpoint the phase behavior of the system shows a number of interesting properties. Some phases may be in equilibrium with excess water solutions and temperature induced transition occurring between phases of different rheology. Monoolein is a nontoxic, biodegradable and biocompatible material classified as GRAS (generally recognized as safe) and it is included in the FDA inactive ingredients guide and in nonparenteral medicines licensed in the United Kingdom. Monoolein show the mesomorphic phase, important in making more comprehensible the potential pharmaceutical application of the lipid. A common feature of lipids is the existence of a temperaturedependent phase transition, below the transition temperature, lipid exist in a gel state. An increase in temperature results in the transition to a fluid like state (liquid crystalline state) similar to the fusion of a crystalline solid. When lipid molecule is heated, instead of melting directly convert into an isotropic liquid. The ability to exist in several different phases is an important property of pure lipids and lipid mixtures; it depends on temperature, hydration and lipid class. In general monoglycerides exhibit different phase behaviors when they exposed to water.

Surfactants, which are used in the production of cubosomes, are poloxamer 407 in a concentration range between $0 \%$ and $20 \% \mathrm{w} / \mathrm{w}$ with respect to the disperse phase. The concentration of the monoglyceride/surfactant mixture generally takes between $2.5 \%$ and $10 \% \mathrm{w} / \mathrm{w}$ with respect to the total weight of the dispersion. Polyvinyl alcohol (PVA) used in addition to poloxamer as a stabilizing agent of the dispersion.

Applications Cubic phase of cubosomes ${ }^{31)}$ is attractive for controlled release because of its small pore size (5$10 \mathrm{~nm}$ ); its ability to solubilize hydrophobic, hydrophilic, and amphiphilic molecules; and its biodegradability by simple enzyme action. ${ }^{32)}$ Cubic phase is strongly bioadhesive ${ }^{33)}$ and is thought to be a skin penetration enhancer ${ }^{34)}$ with excellent compatibility with topical and mucosal deposition and delivery of active ingredients. Recent studies ${ }^{35)}$ have emphasized similarities between the bicontinuous structures formed in human skin layers and those comprising cubic phases, offer- ing the promise of better skin transport understanding and treatment. The tortuous structure of cubic phase tends itself well to slowing diffusive release of solubilized actives. Theory predicts the minimum reduction of a solute's free solution diffusivity by $33 \%$. Commercial applications of cubosomes have been developed for periodontal disease that is based on triglyceride-monoolein mixtures combined with the drug metronidazole. The lipid-drug mixture forms a low-viscosity liquid that, when applied to the gums and placed in contact with saliva, hydrates to form a bulk cubic phase that then delivers the drug to the gum. A short list of applications includes the delivery of actives for periodontal disease ${ }^{36)}$ and implants via in vivo and topical delivery, ${ }^{37)}$ and as bioadhesives. ${ }^{38)}$ Drummond and Fong provide an extensive listing of drug-related applications.

Oral drug Delivery: Cubosomes address the varied challenges in oral delivery of numerous promising compounds including poor aqueous solubility, poor absorption, and large molecular size. These are both liquid and powder in capsule products comprising our self-emulsifying liquid crystalline nanoparticles technology (LCNP), having following advantages:

1) LCNPs are excellent solubilizers, compared with conventional lipid or non-lipid carriers.

2) LCNPs show high drug carrier capacity for a range of sparingly water-soluble drugs.

3) LCNPs are an excellent vehicle to protect the sensitive drug from enzymatic degradation and in-vivo degradation, such as peptides and proteins.

4) The LCNP system enhances the bioavailability range twenty to more than one hundred times of water-soluble peptides.

In an alternative application large proteins have been encapsulated for local activity in the gastrointestinal tract. LCNP carriers can be combined with controlled release and targeting functionalities. The particles are designed to form in situ in a controlled rate, which enables an effective in vivo distribution of the drug. LCNP carriers can also be released at different absorption sites, for example in the upper or lower intestine, which is important for the drugs that have narrow regional absorption window.

Topical Drug Delivery Systems: Topical delivery systems are based on the exploitation of unique properties of liquid crystal (LC) and liquid crystal nanoparticle (LCNP) technologies. Topical drug delivery systems are unique in situ forming bioadhesive LC systems facilitate controlled and effective drug delivery to mucosal surfaces (buccal, ophthalmic, vaginal and others). This fascinating system forms a thin surface film at mucosal surfaces consisting of a liquid crystal matrix which nanostructure can be controlled for achieving an optimal delivery profile and provides good temporary protection of sore and sensitive skin. Their unique solubilizing, encapsulating, transporting and protecting capacity are advantageously exploited in liquid and gel products used to increase transdermal and nasal bioavailability of small molecules and peptides.

Intravenous Drug Delivery Systems: Lipid nanoparticles comprising interior liquid crystal structures of curved lipid membranes are used to solubilize, encapsulate and deliver medications to disease areas within the body. While emulsions and liposomes have found use as intravenous carriers in 
drug products, LCNP structures increased payloads of peptides, proteins and many insoluble small molecules, and are ideal carriers for injection or infusion of many actives.

\section{CONCLUSION}

Cubosomes prepared in dispersions possess a nanometerscale structure identical to bulk cubic phase, but the dispersion itself has much lower, water like viscosity. Compared to liposomes or vesicles, cubosomes possess much higher bilayer area-to-particle volume ratios as well as higher viscous resistance to rupture. Although bulk cubic phase has sufficient length scale to allow controlled release of solutes, cubosomes are too small and have too high a surface area for such performance, exhibiting instead burst release. Other routes may still exist for controlled-release applications of cubosomes e.g. large poly (amidoamine) dendrimer molecules exhibit a $100 \times$ reduction in free diffusivity when entrapped in cubic phases. So the contorted but regular structure of the cubic phases is also of interest to material science researchers as a template for complex solid materials.

\section{REFERENCES AND NOTES}

1) Luzzati V., Tardieu A., Gulik-Kryzwicki T., Rivas E., Riess-Husson F., Nature (London), 220, 485-487 (1968).

2) Scriven L. E., Nature (London), 263, 123-124 (1976).

3) Shah J. C., Sadhale Y., Chilukuri D. M., Adv. Drug Deliv. Rev., 47, $229-230$ (2001).

4) Larsson K., J. Disper. Sci. Technol., 20, 27-34 (1999).

5) Engstrom S., Alfons K., Rasmusson M., Prog. Coll. Pol. Sci., 108, 93-98 (1998).

6) Larsson K., J. Phys. Chem., 93, $7304-7314$ (1989).

7) Spicer P. T., Hayden K. L., Lynch M. L., Langmuir, 17, 5748-5756 (2001).

8) Gustafsson J., Ljusberg-Wahren H., Almgren M., Langmuir, 12, 4611-4613 (1996).

9) Luzzati V., Vargas R., Mariani P., Gulik A., Delacroix H., J. Mol. Biol., 229, 540-551 (1993).

10) Schmidt-Lewerkuhne H., Riedel J. H., Eur. Patent App. EP 0, 67168 A2 (1998).
11) Landau E. M., Rosenbusch J. P., Proc. Natl. Acad. Sci. U.S.A., 93, 14532-14534 (1996).

12) Caboi F., Amico G. S., Pitzalis P., Monduzzi M., Nylander T., Larsson K., Chem. Phys. Lipids, 109, 47-49 (2001).

13) Gustafsson J., Ljusberg-Wahren H., Almgren M., Larsson K., Langmuir, 13, 6964-6971 (1997).

14) Anderson D. M., Wennerstrom H., J. Phys. Chem., 94, 8683-8684 (1990).

15) Luzzati V., Husson F., J. Cell Biol., 12, 207-219 (1962).

16) Fontell K., Mandell L., Ekwall P., Acta Chem. Scand., 22, 3209-3223 (1968).

17) Lutton E. S., J. Am. Oil Chem. Soc., 42, 1068-1070 (1965).

18) Landh T., Larsson K., U.S. Patent No. 5531925 (1996).

19) Siekmann B., Bunjes H., Koch M. H. J., Westesen K., Int. J. Pharm., 244, 33-43 (2002).

20) Nakano M., Sugita A., Matsuoka H., Handa T., Langmuir, 17, 3917 3922 (2001).

21) Radiman S., Toprakcioglu C., Mcleish T., Langmuir, 10, 61-67 (1994).

22) Almgren M., Edwards K., Gustafsson J., Curr. Opin. Colloid. Interface Sci., 1, 270-278 (1996).

23) Siekmann B., Bunjes H., Koch M. H. J., Int. J. Pharm., 244, 33-43 (2002).

24) Spicer P. T., Small W. B., Lynch M. L., Burns J. L., J. Nanopart. Res., 4, 297-311 (2002).

25) Strom P., Anderson D. M., Langmuir, 8, 691-709 (1992).

26) Lynch M. L., Kochvar K. K., Burns J. L., Laughlin R. G., Langmuir, 16, 3537-3542 (2000).

27) Engström S., Larsson K., Lindman B., Controlled Release Bioact. Mater., 15, 105-106 (1998).

28) Engström S., Lindahl L., Wallin R., Engblom J., Int. J. Pharm., 86, 137-145 (1992)

29) Boyle E., German J. B., Crit. Rev. Food Sci. Nutr., 36, 785-805 (1996).

30) Rosevear F. B., J. Am. Oil Chem. Soc., 31, 628-639 (1954).

31) Drummond C. J., Fong C., Curr. Opin. Colloid. Interface Sci., 4, 449-456 (2000).

32) Borne J., Nylander T., Khan A., J. Phys. Chem. B, 106, 10492-10500 (2002).

33) Geraghty P. B., Attwood D., Collett J. H., Sharma H., Dandiker Y., Biomaterials, 18, 63-67 (1997).

34) Lee J., Kellaway I. W., Int. J. Pharm., 204, 137-144 (2000).

35) Norlen L., J. Invest. Dermatol., 117, 823-829 (2001).

36) Czarnecki R. F., Williams D. L., U.S. Patent 5230895 (1993).

37) Andersson S., Jonn S., Landh T., W.O. Patent 991517 (1999).

38) Hansen J., Nielsen L. S., Norling T., U.S. Patent 5955502 (1999). 